\title{
The Big Bang and its Dark-Matter Content: Whence, Whither, and Wherefore
}

\author{
Roger Penrose ${ }^{1}$
}

Received: 20 March 2018 / Accepted: 27 March 2018 / Published online: 16 June 2018

(C) The Author(s) 2018

\begin{abstract}
The singularity theorems of the 1960s showed that Lemaitre's initial symmetry assumptions were not essential for deriving a big-bang origin for a vast multitude of relativistic universe models. Yet the actual universe accords remarkably closely with models of Lemaitre's type. This is a mystery closely related to the form taken by the 2nd law of thermodynamics and is not explained by currently conventional inflationary cosmology. Conformal cyclic cosmology (CCC) provides another perspective on these issues, one consequence being the necessary initial presence of a dominant scalar material that interacts only gravitationally, but which must ultimately slowly decay away in a novel but perhaps detectable way. According to CCC, our current universe picture provides but one aeon of an unending succession of expanding aeons each having an initial big bang which is the conformal continuation of the remote exponential expansion of its previous aeon. The observational status of CCC is briefly discussed.
\end{abstract}

Keywords Singularity theorems $\cdot$ Conformal cosmology $\cdot$ Dark matter

\section{Lemaître's Big Bang and the Singularity Theorems}

Georges Lemaître made a huge contribution to cosmology when he pointed out that Einstein's equations, in a cosmological setting, in which the galaxies expand away from each other (the observational evidence for which he was well aware of) led one to believe that the universe could well have had a singular origin, a finite time ago. However, Lemaître's conclusions, along with those of all other prominent cosmologists of the time, were based on the assumption that the universe can be treated as

Roger Penrose

rpenroad@gmail.com

1 Mathematical Institute, Oxford, UK 
being spatially homogeneous and isotropic. This assumption seemed reasonably well supported, on the observational side, but more importantly, it was an assumption that allowed Einstein's equations to be solved explicitly, so that he, Friedmann, and others could supply neat explicit solutions of Einstein's equations.

Of course, the actual universe is not exactly of this completely symmetrical nature, the mere presence of separate galaxies each containing myriads of separated stars implies that the mass distribution, and therefore the Ricci-tensor distribution is, in detail very far from uniform. Nevertheless, it is a natural expectation, as we evolve Einstein's equations into the future, that the overall picture will remain well approximated by the exact Friedmann-Lemaitre-type model. This is despite the fact that at the very local level, the development of black holes, in particular, would give us something greatly differing from the smoothed-out overall picture. These local deviations from the overall picture are to be expected, but are not regarded as invalidating the usefulness of this Friedmann-Lemaitre-Robertson-Walker (FLRW) picture.

All this notwithstanding, the situation is very different when we consider the evolution of Einstein's equations into the past. It is useful to think of this in time-reversed terms, where we imagine a universe model that is collapsing. In such a situation, we expect density irregularities to increase as the universe contracts, and the FLRW approximation would get worse and worse. In the late stages of the collapse, these irregularities would result in numerous black holes. Time reversing this picture (and taking into account the invariance of Einstein's equations under time-reversal), we find that the singular origin that we find in the FLRW models is something extremely special.

Indeed, the very existence of the initial singularity, under such generic circumstances had often been questioned. Perhaps, in the generic case there might be a complicated kind of non-singular "bounce", where the early expansion might have resulted from a highly irregular previous collapsing phase (see Fig. 1). However, it turns out that such non-singular bounces are inconsistent with certain very basic physical assumptions about energy positivity in the matter density, etc., as was shown in various singularity theorems proved mainly in the $1960 \mathrm{~s}$. In the first of these [1], I showed, by using techniques involving differential topology, that in gravitational collapse to what we now call a black hole, the assumptions of exact spherical symmetry and a pressure-less fluid ("dust"), that Oppenheimer and Snyder had made [2], in their picture of collapse to a black hole could be dispensed with, and collapse to a space-time singularity remained a consequence of Einstein's theory even in a completely generic collapse. Soon afterwards, Stephen Hawking showed [3] how my theorem could be used (in the reverse time direction) to obtain a singularity theorem that applied to the universe a whole. He then made a study of the appropriate mathematical techniques (Morse theory, modified so as to be applicable in Lorentzian, rather than Riemannian manifolds) and he wrote a series of papers that were published in the Proceedings of the Royal Society of London [4-6] (having had some help from Brandon Carter in the removal of some initial minor errors). Finally, in 1970, a joint paper between Hawking and myself appeared in that journal [7], which generalized the results that had been obtained before in a very comprehensive result, showing, in particular, that a singularity-free "bounce", of the nature of that illustrated in Fig. 1, cannot occur with 
Fig. 1 An imagined situation in which a collapsing universe model "bounces" in a non-singular way to an expanding phase, via some complicated intermediary state. The singularity theorems rule out this behavior in classical general relativity, with reasonable physical restrictions on the matter density

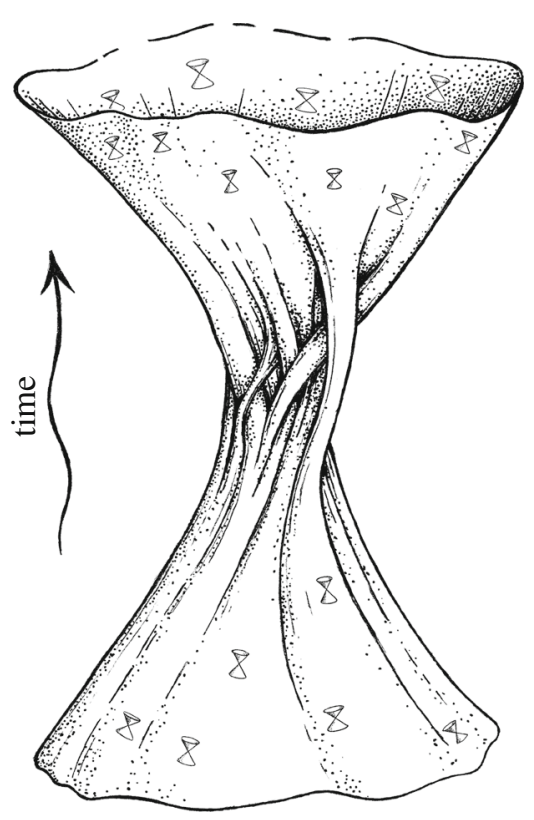

any type of matter source with stress-energy tensor satisfying a very general condition of local energy non-negativity.

\section{The Second Law of Thermodynamics, and its Remarkable Coherence with Lemaître's Assumptions}

For a while I had found it puzzling that cosmologists did not seem to take seriously the vast multitude of anisotropic models for the early universe, with initial singularities that bore little similarity to those of the FLRW models. I recall mentioning my puzzlement to James Peebles, who commented to me the good reason that such isotropy as is exhibited by FLRW models is in remarkable agreement with what is observed, particularly in the cosmic microwave background (CMB). This fact, I had not properly appreciated before, and it immediately set me on a line of thinking that has deeply concerned me ever since (see, for example [8-12]). This has to do with the second law of thermodynamics (henceforth abbreviated "2nd Law"), in a cosmological context. The usual statement of the 2nd Law, that there is a near-universal tendency of the entropy of a system to increase into the future, can be equally be stated in the past direction, namely that there is such a universal tendency to decrease as we examine things further and further into the past. Accordingly, the situation at the Big Bang ought to have been one of exceptionally low entropy. This expectation may be contrasted with the most direct observational evidence of the early universe, as provided by the CMB, namely its virtually perfect Planck spectrum [13], which would appear to be indicative of a maximum entropy state! 
This apparent contradiction is explained by the fact that the evidence from the CMB comes from photons and their interactions with matter. This Planck spectrum says nothing about the gravitational field. The uniformity over the sky of the CMB-with temperature variations of only around one part in $10^{5}$ (once the Doppler effect due to the Earth's proper motion is corrected for) - is strongly indicative of a highly uniform matter distribution, and strong concordance with the standard FLRW picture. The important thing to realize, here, is that with regard to the gravitational field, the uniform state is of exceedingly low entropy, owing to gravity's universally attractive nature. In contrast with the behaviour of a gas in a box, for example, where maximum entropy would be pictured as something with great spatial uniformity, gravitating bodies, such as systems of stars, would tend to clump more and more in their spatial distribution, as their dynamical time evolution proceeds, representing an increase in the gravitational entropy. The greatest clumping of all occurs with the formation of black holes, which would be accompanied by an absolutely enormous increase in the entropy.

Thus, the early universe, at the time of the emission of the CMB radiation (at around 380,000 years after the Big Bang), was very curiously imbalanced with regard to its entropy. It appears to be the case that the gravitational degrees of freedom were hardly activated at all at that time, but that the matter and radiation were in a maximum entropy state. The situation at even earlier times cannot have been any less imbalanced, in this regard, as follows simply from general thermodynamic principles. This applies also to the action of inflation, despite the fact that it is often argued that a very early exponential inflationary expansion would serve to iron out any spatial irregularities in the initial geometry of the universe. It may be seen, by considering a time-reversed collapsing universe that for a generic (non FLRW) Big Bang, the presence of an inflaton field is of no help whatever in smoothing out such an irregular initial singular expansion (see [14] for a more thorough explanation of this point).

In a general way, it is not hard to see how the early low gravitational entropy of our universe, as exhibited in its spatial uniformity, eventually gave rise to the low-entropy structures that we are familiar with. We must admit to there having been some initial small density irregularities in the early universe, these-by means of whatever subtle processes might have been involved-eventually contracted into stars and planets. The planet we are most familiar with is, of course, the Earth, and on it we witness innumerable examples of low-entropy living structures. Almost all of these depend upon the low-entropy environment of a hot localized region of the sky that we recognize as the Sun, in contrast with the cold dark night sky, the temperature imbalance between the two being what drives the low-entropy life on Earth $[8,15,16]$.

Not only was the lowness in our universe's initial entropy almost entirely in the suppression of gravitational degrees of freedom but it is in gravity that we see the very hugeness of the entropy that is potentially present in the universe, as is demonstrated by the Bekenstein-Hawking formula [17, 18] for the entropy in a black hole. Even by now, at the present stage of the universe/s evolution, there is by a huge factor far more entropy in supermassive black holes in galactic centres than in all other sources of entropy put together. In making this comment I should make clear that we cannot regard black-hole entropy as something thermodynamically different from other forms of entropy. For full consistency with the 2nd Law, the black-hole entropy must be taken 
into account [19]. To estimate the total entropy potentially available in our universe, for a matter content equal to that currently within our particle horizon (including the dark matter), we may think of a black hole of mass equal to that total matter content. This entropy is of the general order of $10^{124}$ [16]. Taking this to be the maximum entropy available for that body of matter, and regarding this entropy in the Boltzmannian sense of the logarithm of, essentially, the total phase-space volume available, we find this volume to be of the rough order of

$$
10^{10^{124}}
$$

in any reasonable units (see [12], Chap. 3, for example). This figure gives us some idea of the vast improbability of our Big Bang (with gravitational degrees of freedom being effectively set to zero), had it been selected randomly.

To me, this is an extraordinary fact about universe, and I find it very puzzling that cosmologists do not tend to list it among the fundamental mysteries of cosmology that need to be resolved. As mentioned above, this mystery is in no way explained by the assumption of an early inflationary phase to the universe [14]. In fact, to my knowledge, the only cosmological scheme that does provide an explanation is conformal cyclic cosmology (or CCC). This proposal I come to next.

\section{The Ideas of Conformal Cyclic Cosmology}

According to CCC $[12,16,20-22]$, the universe consists of a (perhaps infinite) succession of aeons, where each aeon originates with its own big bang and has an unending exponentially expanding future, consistent with a positive cosmological constant $\Lambda$. We take $\Lambda$ to have the same constant value from aeon to aeon, the Einstein $\Lambda$-equations holding throughout every aeon. The remote future of each aeon matches conformally the big bang ${ }^{1}$ of the next one (see Fig. 2), so that the conformal space-time geometry is smooth across the join, which is a space-like hypersurface referred to as the crossover 3-surface.

The driving idea behind CCC is the 2nd Law, in the particular form that it appears to have been presented to us by nature, as described in $\S 2$, namely that the initial extremely low entropy at the beginning of each aeon is manifested in the fact that although the matter and radiation are pretty-well thermalized immediately following the big bang of each aeon, the gravitational degrees of freedom are taken to be completely suppressed at its big bang, so that the Weyl tensor $C_{a b c d}$ vanishes there. In CCC, this "Weyl curvature hypothesis" (see e.g. [11, 12]) is replaced by Paul Tod's proposal [23] that each aeon's big bang can be smoothly conformally extended backwards into another space-time region. That region is, according to $\mathrm{CCC}$, taken to be the remote future (conformal infinity) of a previous aeon, the crossover 3-surface being that earlier aeon's conformal infinity [12, 16, 24-26].

\footnotetext{
${ }^{1}$ I use the capitalized "Big Bang" for the specific occurrence that initiated our particular aeon, but without capitalization for the general concept.
} 

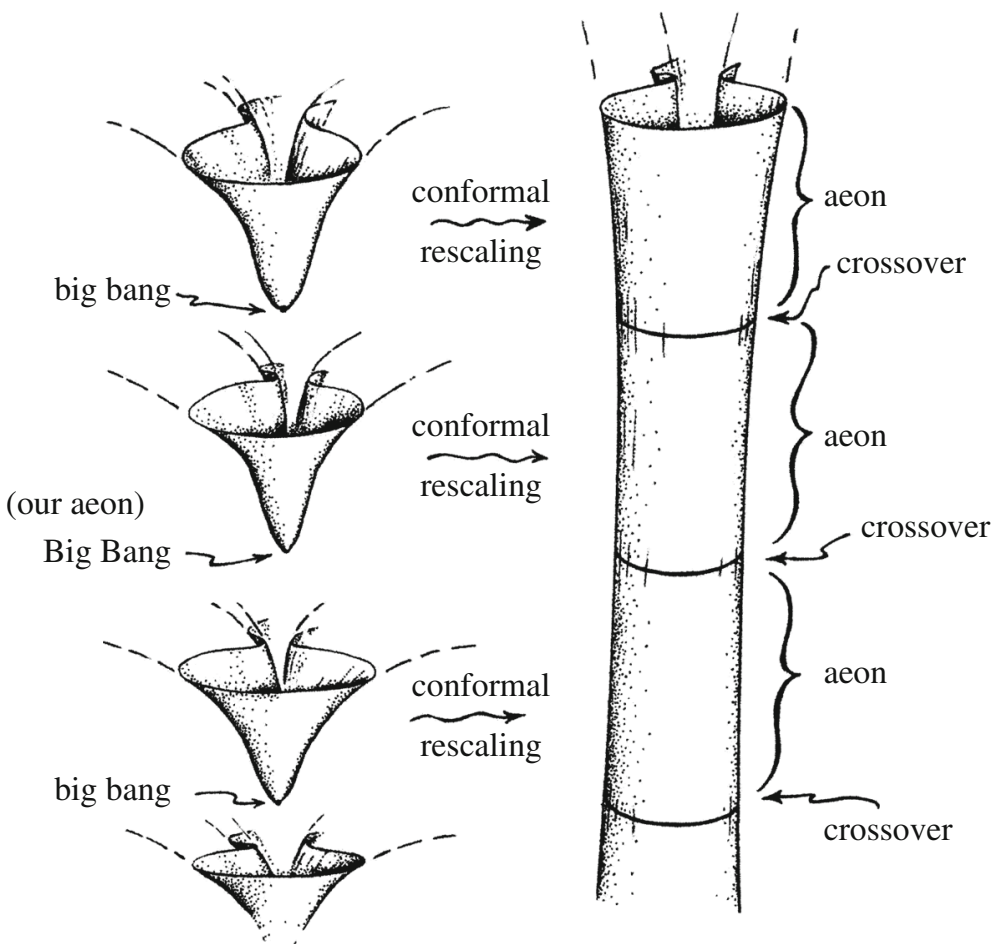

Fig. 2 Schematic description of conformal cyclic cosmology (CCC). The picture is drawn so as to allow for the spatial geometries to be either all open, or all closed

There is a theorem, due to Helmut Friedrich [27] that tells us that, with positive $\Lambda$ and electromagnetism (or other massless fields) as the matter content, a smooth space-like conformal future infinity will be a generic situation, so that the conformal continuation of the remote future of one aeon to some other space-time is a generic possibility. But according to $\mathrm{CCC}$, we take this continuation to be to the big bang of a further aeon. As it stands, this is merely a vaguely plausible geometrical picture, and it may be difficult to take it seriously as a physically acceptable model. However, when examined from the physical point of view, it does indeed hang together in a remarkably plausible way.

The physics near the big bang is at an enormous temperature, so rest-mass is completely dominated by kinetic motions, and so may be ignored. Without mass, the physics may plausibly be considered to be dominated by conformally invariant processes, as will be considered in a little more detail shortly. Moreover our current picture of the distant future of the universe, according to Einstein's $\Lambda$-equations is a continuing exponential expansion, with matter density continually reducing and an entropy content completely dominated by supermassive black holes. Eventually (after some $10^{100}$ years or so), even these supermassive black holes will have all evaporated away by Hawking radiation, to leave an exceedingly rarefied universe whose particle number content will be almost entirely in the form of photons, so the conditions of Friedrichs's theorem are basically satisfied. There will, however, be a residue of a 
relatively small number of massive particles, and for the strict requirements of the theory one must apparently assume that there is a mechanism whereby in the hugely long-term future, mass eventually fades away, asymptotically-which I shall refer to as "mass fade-out" — an admittedly unconventional hypothesis, but not contradicted by current observation, and not implausible on the basis of a particle physics which is extended from current ideas by the presence of a positive $\Lambda$ (perhaps by virtue of the fact that, unlike for the Poincaré group, mass is not a Casimir operator for the de Sitter group).

Is it physically plausible that this enormously rarefied and absurdly cold future infinity can be physically similar to the enormously dense and hot big bang of a succeeding aeon? Yes, indeed, if we may regard the relevant physics to be a conformally invariant one, where we bear in mind that not only are the equations of Maxwell conformally invariant, but so also are those of Yang-Mills, when mass can be ignored (see, for example [28]). This conformal freedom allows us to stretch out the hot big bang of the succeeding aeon and to squash down the remote future of the previous one-bearing in mind that energy and momentum scale in the exact inverse way to space and time. So hot becomes cold and dense becomes rarefied upon conformal stretching [16].

An explanation is required concerning the 2 nd Law, as one might consider this problematic in a cyclic model. CCC's response is, first, to take note of the fact that, as remarked earlier, even now, by far the major contribution to the entropy in the universe is in supermassive black holes. This will vastly increase in our aeon's future. But ultimately, by Hawking radiation, all black holes will disappear via Hawking evaporation. The issue here is that, in CCC, one must side with those who believe that information - or, more to the point, phase-space volume - must be destroyed by being obliterated at the hole's singularity. As has often been pointed out, this is in contradiction with quantum unitarity, but the view here is taken (in opposition to that of "many-worlds" proponents) that since unitarity is already violated in quantum measurement, then it is not a universal truth; moreover, the viewpoint will be taken here that in quantum measurement as well as with black-hole evaporation, quantum state reduction is a gravitational effect (see, for example [29-33]). Thus, the view taken in CCC is that when a black hole has eventually evaporated away, many degrees of freedom will have been removed from the system (namely those describing whatever had been destroyed at the black hole's singularity), so that the phase space that now effectively describes the state of the universe has become significantly reduced, and accordingly the effective entropy describing the system is now defined in terms of a smaller phase space, namely that for which the degrees of freedom swallowed by the hole are removed from consideration. The 2 nd Law is not violated, but the entropy definition that is of relevant to the system now, after the black hole is gone, has a value smaller than the one previously. See $[12,16]$. When all black holes have evaporated away, at the crossover 3-surface, the effective entropy will have dropped to the very low value that is required to start off the next aeon. Thus, the 2 nd Law is not violated; it is transcended in the sense that the effective entropy definition has to shift down to that which is relevant to the new aeon. 


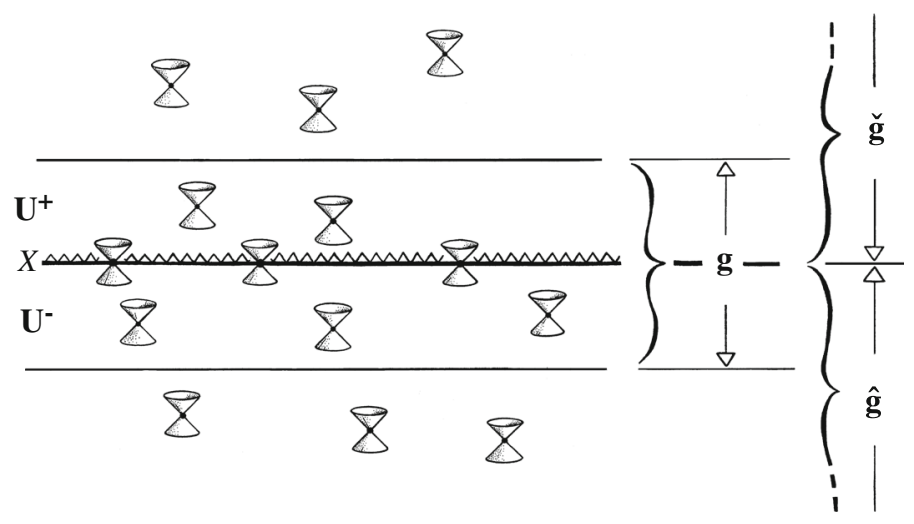

Fig. 3 Picture of the bandage region joining the remote future of one aeon to the big bang of the next

\section{The Equations of $\mathrm{CCC}$}

Of course one needs clear-cut equations at crossover to describe this transition from the remote future of one aeon to the big bang of the next. Such equations can be provided (see [22], Appendix A [34]), although there are still uncertainties, in some detailed choices for ensuring uniqueness of evolution, these will not concern us here. Nevertheless, there are major issues concerning this evolution that are central to the current discussion.

The transition from each aeon to the next is described in terms of a "bandage metric" $g_{a b}$ that is defined throughout an open region $U$ containing the crossover hypersurface $X$ that connects the two aeons under consideration. See Fig. 3. Here $U^{-}$represents the pre-crossover part of $U$ and $U^{+}$, the post-crossover part. Let $\hat{g}_{a b}$ be the physical metric for the aeon pre-crossover and $\check{g}_{a b}$, the physical metric post-crossover. We have conformal factors $\Omega$ and $\omega$ so that

$$
\hat{g}_{a b}=\Omega^{2} g_{a b} \text { and } \check{g}_{a b},=\omega^{2} g_{a b}
$$

where the reciprocal hypothesis

$$
\Omega=-\omega^{-1}
$$

is taken to hold throughout $U$, and where $\omega$ is smooth, changing from negative in $U^{-}$to positive in $U^{+}$, being zero on $X$. Thus, $\Omega$ is infinite on $X$, positive in $U^{-}$and negative in $U^{+}$. representing a "squashing down" of the remote future of the earlier aeon so as to fit smoothly on to the "stretched out" big bang of the subsequent aeon, the stretching being provided by $\omega$. The crossover 3 -surface $X$, is defined by $\omega=0$.

Within the earlier aeon, the $\Omega$-field has no physical content, but we can take it as a kind of auxiliary field—a kind of "phantom" or "gauge" field—which allows us to express Einstein's equations in a conformally invariant framework, and when taken alongside the gravitational field (described by $\hat{C}_{a b c d}$ ) allows us to extend the range of Einstein's theory so that it includes the crossover 3 -surface $X$. There are no additional 
physical degrees of freedom in $\Omega$, beyond those already in the gravitational field. Yet it has an "energy tensor", which is

$$
T_{a b}[\Omega]=\frac{1}{4 \pi G} \Omega^{3}\left\{\nabla_{a} \nabla_{b}-\frac{1}{4} g_{a b} \nabla^{c} \nabla_{c}-\frac{1}{2} R_{a b}+\frac{1}{8} R g_{a b}\right\} \Omega^{-1}
$$

which is trace-free and divergence-free [35], 2nd footnote on p. 194 (see also [36]). The Einstein $\Lambda$-equations then take the remarkably simple conformally invariant form

$$
T_{a b}=T_{a b}[\Omega]
$$

where $T_{a b}$ is the energy tensor of the matter, scaling according to

$$
\hat{T}_{a b}=\Omega^{-1} T_{a b}
$$

(the matter being assumed to be massless-where it is taken that the assumption of mass fade-out, referred to in Sect. 3, has effectively killed off all rest-mass by the time that $U^{-}$is reached, so that $T_{a b}$ may be assumed trace-free within $U^{-}$).

However, on the future side of $X$, in $U^{+}$, when the $\check{g}_{a b}$ metric is used, the $\Omega$ field behaves as an actual physical field. This is a consistent viewpoint because with the reciprocal hypothesis holding, $\Omega$ behaves as an ordinary scalar field, under further conformal rescaling ([22] Appendix A). For this field to be uniquely defined, we require certain conditions to be imposed on the first few normal derivatives of $\omega$ at $X$. (See [22] Appendix A and [34]; the issue is somewhat delicate, and various alternatives are possible, but the details will not concern us here.). This scalar field starts off as effectively massless, but would rapidly acquire a mass and would be expected to dominate over all other fields just following the big bang. This is a necessary feature of CCC because for Einstein's equations to hold on both sides of $X$, we need a huge matter density post-crossover that was not present pre-crossover.

It is a hypothesis of CCC that this $\Omega$-field, within $U^{+}$, when it acquires a mass, is in fact the dark-matter field that provides the dominant matter content of the universe (where $\Lambda$, or whatever else "dark energy" might be, does not count as "matter"). We shall come to this in more detail in Sect. 5. The gravitational degrees of freedom in the earlier aeon may be considered to be described by the Weyl conformal tensor $\hat{C}_{a b c d}$. However, we find, with the conformal curvature scaling as

$$
\hat{C}_{a b c d}=\Omega^{2} C_{a b c d}
$$

(see [37] Chap. 9), that

$$
C_{a b c d}=0 \text { at } X
$$

which is in accordance with the Weyl curvature hypothesis [10-12, 23] the conformal curvature matching from aeon to aeon across $X$. Nevertheless, gravitational wave signals in that earlier aeon do register at $X$ in the normal derivative of $C_{a b c d}$ across $X$. This information is not lost at crossover, but part of it (the "electric" part of this normal 
derivative) is conveyed as 4 th derivatives of the $\Omega$-field (the "magnetic" part being encoded into an intrinsic conformal curvature-the Cotton tensor-of the 3-space $X$ (first pointed out by Paul Tod [34]).

Such signals would affect the CMB of the succeeding aeon in observable ways. Up until now, observational interest, concerning CCC, has been concentrated on the prospect of observing, in our current aeon, the effect of supermassive black-hole encounters in the aeon prior to ours. The remote future of the emitted gravitational waves should, according to CCC, register in the CMB of our aeon, as circular features, sometimes in concentric sets (indicating several such events occurring, at various times, in the same galactic cluster in the previous aeon), and strong arguments have been put forward that such signals are indeed seen in the WMAP data [22,38], compare [39], and also in the Planck data [40, 41]. It may be remarked that these observations demonstrate that the sources of these signals have a highly inhomogeneous distribution across the sky, which is consistent with CCC, but highly problematic for the conventional explanation in terms of quantum fluctuations in an inflaton field.

\section{Dark Matter and its Decay, According to CCC}

Of more relevance to the present discussion are the almost scale-invariant temperature fluctuations in the CMB. This scale invariance is one of the strongest motivations for a belief in an exponentially expanding inflationary phase in our very early universe. In CCC, there should not be any such inflationary phase in our aeon, because that would magnify the conformal gap between the previous aeon and the last-scattering 3 -surface that generates our CMB, to an enormous degree, and would render the relationship between these two aeons to be extremely remote. Moreover, the circular features referred to above would not be seen at all in the way that observation indicates. Instead, in CCC, the role of inflation is taken over by the exponentially expanding ultimate history of the previous aeon. In a sense, "inflation" of a sort does take place in CCC, but it occurs "before", rather than after the Big Bang (echoing an earlier idea expressed by Veneziano and Gasperini [42]).

For the exponential expansion of the remote future of the previous aeon to provide the near scale-invariant CMB temperature fluctuations that are observed, we need something to take over the role of the quantum fluctuations of an inflaton field, which are demanded by conventional $\Lambda$ CDM theory. Thus, in CCC, we need an appropriate source for the CMB temperature fluctuations, which would have to inhabit the exponentially expanding phase of the previous aeon in a spatially very uniformly distributed way. The most plausible candidate would appear to be the decay of the dark-matter particles which, as already remarked in Sect. 4, would come about according to CCC as the quantum constituents of the $\Omega$-field that dominates the matter content of the universe immediately after crossover. I call these dark-matter particles erebons, after Erebos, the primordial ancient Greek god of darkness. Since the erebons need to be created afresh, according to $\mathrm{CCC}$, at the beginning of each aeon, they must decay completely during the course of each aeon. It is the erebon decay that CCC proposes to be the source of the CMB temperature fluctuations the succeeding aeon. 
Erebons would have to be rather unusual particles. They are simply the product of gravitation alone, according to CCC, and would interact only gravitationally. Since no other physical interactions (electromagnetic, weak, or strong) are involved, their mass would have to be something of the order of a Planck mass $\left(10^{-5} \mathrm{~g}\right)$ or thereabouts, perhaps with some factor such as $8 \pi$ involved. From the point of view of standard Poincaré-invariant particle physics, erebons would be completely stable particles, but, in the presence of a cosmological constant $\Lambda$, erebons should be able to decay, with a lifetime of perhaps something like $10^{11}$ years, in my estimation, and could be the cause of the (near) scale-invariant CMB temperature fluctuations in the following aeon, according to $\mathrm{CCC}$. The inflations of conventional $\Lambda \mathrm{CDM}$ theory cannot be playing this role in CCC, since there is no inflation in CCC. Instead, in CCC, it is the erebon decay in the previous aeon that takes over the inflations' role.

There could be an important technical advantage in this kind of idea, over that of the conventional $\Lambda \mathrm{CDM}$ theory. To my mind, there has always been a serious though normally ignored issue, of how a quantum fluctuation effect could be the source of such manifestly classical large-scale phenomenon as the CMB temperature variations. If, indeed, erebon decay is responsible, as CCC seems to demand, then we must take into account the erebon mass which, though enormous from the point of view of standard particle physics, is well within the range of possibilities allowed by astrophysical observation [43]. The issue of quantum state reduction-i.e. how it is that quantum amplitudes seem to get converted into measures describing the various probabilities of the occurrences of different classical alternatives-is highly fraught and contentious. However, there is a general point of view that I strongly favour myself, that it is gravity which effects the physical reduction of a quantum state into classical alternatives [28-30, 33, 38, 44-46]. Although the different schemes of this kind differ somewhat from each other, there is the general expectation that within such proposals the quantum state of a Planck-mass particle ought to be continually self-reducing, so that, individually it ought to behave very like a classical particle, and its decay may be regarded as a classical event. Accordingly, if such decays in the aeon prior to ours could make impressions on the behaviour of matter in the early stages of our own aeon, these ought already to be classical events, so that the aforementioned issue concerning the "classicalization" of quantum fluctuations in the inflaton field should be resolved in the CCC scheme.

We still have to answer, in this CCC picture, the question: what must be expected as the product of an erebon decay? Again, according to CCC, erebons are entirely gravitational entities, interacting only gravitationally, so their decay ought to be entirely into gravitons. But, again the classical/quantum issue looms large, and the CCC expectation is that their decay ought to be into something effectively in accordance with classical general relativity. Yet, some quantum issues still seem to retain their importance. One of these is that although the state-reduction of individual erebons may render them to behave individually like classical particles, their bosonic character (arising from their being scalar particles) may well influence the statistics of what is observed with large numbers of erebons and their decays. Another (perhaps related) issue of erebon decay would be that, along with their Planck mass, there ought to be a Planck frequency involved in their decay. 
The way that I envisage that such a decay could be described, is as an extremely high frequency gravitational wave. According to an early discussion of mine [47], such a wave would appear, at larger scales far greater than the wavelength, like a wave of zero-rest-mass matter. Thus, erebon decay could be closely modelled by a Vaidya solution [48], where the initial state of a massive particle described by a Schwarzschild metric decays into scalar radiation. A much more thorough discussion of such waves has recently been carried out by Cécile Heneau and Jonathan Luk [49] which provides a complete description of such high-frequency gravitational fields in terms of a massless scalar field. It may therefore be appropriate to model erebon decay in terms of impulsive waves of such a scalar field.

There is an intriguing issue concerning whether such erebon decays in our own aeon might be detectable by gravitational wave detectors such as LIGO. It is even conceivable that there may be hints of such signals (from distant galaxies) in the "noise" surrounding black-hole encounters as detected by LIGO [50]. Perhaps, instead, erebon decay will need detectors of a different kind [51,52] to see erebon decays in our aeon, if such decays actually do exist, of the kind described here.

Acknowledgements My thanks go to Iwo Bialynicki-Birula, Paul Tod, and most particularly to Krzysztof Meissner, discussions with whom led to the idea that dark-matter decay in the previous aeon might be responsible for the near scale invariant $\mathrm{CMB}$ temperature fluctuations, and to Ivette Fuentes, with whom discussions of possible detection of such decay in our own aeon using her proposed BEC detectors led me to the unusual line of thinking suggested here. I am also grateful for financial assistance through a personal endowment from J.P. Moussouris.

Open Access This article is distributed under the terms of the Creative Commons Attribution 4.0 International License (http://creativecommons.org/licenses/by/4.0/), which permits unrestricted use, distribution, and reproduction in any medium, provided you give appropriate credit to the original author(s) and the source, provide a link to the Creative Commons license, and indicate if changes were made.

\section{References}

1. Penrose, R.: Gravitational collapse and space-time singularities. Phys. Rev. Lett. 14, 57-59 (1965)

2. Oppenheimer, J.R., Snyder, H.: On continued gravitational contraction. Phys. Rev. 56, 455-459 (1939)

3. Hawking, S.W.: Occurrence of singularities in open universes. Phys. Rev. Lett. 15, 689 (1965)

4. Hawking, S.W.: The occurrence of singularities in cosmology. Proc. Roy. Soc. Lond. A 294, 511-521 (1966)

5. Hawking, S.W.: The occurrence of singularities in cosmology II. Proc. Roy. Soc. Lond. A 295, 490 (1966)

6. Hawking, S.W.: The occurrence of singularities in cosmology III. Causality and singularities. Proc. Roy. Soc. Lond. A 300, 187-201 (1967)

7. Hawking, S.W., Penrose, R.: The singularities of gravitational collapse and cosmology. Proc. Roy. Soc. Lond. A 314(1519), 529-548 (1970)

8. Penrose, R.: The Emperor's New Mind: Concerning Computers, Minds, and the Laws of Physics, Oxford Univ. Press, Oxford. ISBN: 0-19-851973-7 (1989)

9. Penrose, R.: Singularities and Time-Asymmetry. In: Hawking, S.W., Israel, W. (eds.) General Relativiy: An Einstein Centenary. Cambridge University Press, Cambridge (1979)

10. Penrose, R.: Singularities of space-time. In: Lebowitz, N.R., Reid, W.H., Vandervoort, P.O. (eds.) Theoretical Principles in Astrophysics and Relativity. Chicago University Press, Chicago (1978)

11. Penrose, R.: The Road to Reality: A Complete Guide to the Laws of the Universe. Jonathan Cape, London. Vintage IBN: 9780-679-77631-4 (2004)

12. Penrose, R.: Fashion, Faith, and Fantasy in the New Physics of the Universe. Princeton University Press, Princeton (2017) 
13. Liddle, A.R.: An Introduction to Modern Cosmology. Wiley, Chichester (1999)

14. Penrose, R.: Difficulties with inflationary cosmology. In: Fenves, E. (ed.) Proceedings of the 14th Texas Symposium on Relativistic Astrophysics. N.Y. Acad. Sci., New York (1990)

15. Schrödinger, E.: What is Life?' and 'Mind and Matter. Cambridge Univ. Press, Cambridge (1967)

16. Penrose, R.: Cycles of Time: An Extraordinary New View of the Universe. Bodley Head, London. ISBN 978-0-224-08036-1; 9780099505945 (2010)

17. Bekenstein, J.: Black holes and entropy. Phys. Rev. D 7, 2333-2346 (1973)

18. Hawking, S.W.: Black holes and thermodynamics. Phys. Rev. D 13(2), 191 (1976)

19. Unruh, W.G., Wald, R.M.: Entropy bounds, acceleration radiation, and the generalized second law. Phys. Rev. D 27, 2271 (1982)

20. Penrose, R.: Before the big bang: an outrageous new perspective and its implications for particle physics. In: Prior C.R. (ed.) EPAC 2006-Proceedings, Edinburgh, Scotland, pp. 2759-2762. European Physical Society Accelerator Group, EPS-AG (2006)

21. Penrose, R.: Causality, quantum theory and cosmology. In: Majid, S. (ed.) On Space and Time, pp. 141-195. Cambridge University Press, Cambridge. ISBN 978-0-521-88926-1 (2008)

22. Gurzadyan, V.G., Penrose, R.: On CCC-predicted concentric low-variance circles in the CMB sky. Eur. Phys. J. Plus 128, 22 (2013). https://doi.org/10.1140/epjp/i2013-13022-4

23. Tod, K.P.: Isotropic cosmological singularities: other matter models. Class. Quantum Grav. 20, 521-534 (2003). https://doi.org/10.1088/0264-9381/20/3/309

24. Penrose, R.: Asymptotic properties of fields and space-times. Phys. Rev. Lett. 10, 66-68 (1963)

25. Penrose, R.: Conformal approach to infinity. In: DeWitt, B.S., DeWitt, C.M. (eds.) Relativity, Groups and Topology: The 1963 Les Houches Lectures. Gordon and Breach, New York (1964)

26. Penrose, R.: Zero rest-mass fields including gravitation: asymptotic behaviour. Proc. Roy. Soc. Lond. A 284, 159-203 (1965)

27. Friedrich, H.: Einstein's equation and conformal structure. In: Huggett, S.A., Mason, L.J., Tod, K.P., Tsou, S.T., Woodhouse, N.M.J. (eds.) The Geometric Universe; Science, Geometry, and the Work of Roger Penrose. Oxford Univ. Press, Oxford (1998)

28. Penose, R., Rindler, W.: Spinors and Space-Time: Two-Spinor Calculus and Relativistic Fields, vol. 1. Cambridge University Press, Cambridge (1984)

29. Diósi, L.: Gravitation and quantum-mechanical localization of macro-objects, 105A, 199-202; (1987) A universal master equation for the gravitational violation of quantum mechanics. Phys. Lett. A 105, 199-202 (1984)

30. Diósi, L.: A universal master equation for the gravitational violation of quantum mechanics. Phys. Lett. A 120, 377-381 (1987)

31. Penrose, R.: On gravity's role in quantum state reduction. Gen. Rel. Grav. 28, 581-600 (1996)

32. Penrose, R.: On the gravitization of quantum mechanics 1: quantum state reduction. Found. Phys. 44, 557-575 (2014)

33. Fuentes, I., Penrose, R.: Quantum state reduction via gravity, and possible tests using Bose-Einstein condensates. In: Gao, S. (ed.) Collapse of the Wave Function: Models, Ontology, Origin, and Implications. Cambridge Univ. Press, Cambridge (2018)

34. Szabados, L.B., Tod, K.P.: A positive Bondi-type mass in asymptotically de Sitter spacetimes. Class. Quantum Gravity 32, 205011 (2015)

35. Newman, E.T., Penrose, R.: New conservation laws for zero rest-mass fields in asymptotically flat space-time. Proc. Roy. Soc. Lond. A 305, 175-204 (1968)

36. Bramson, B.D.: On the role of conformal invariance in General Relativity and the origin of mass. Phys. Lett. 47A, 431-432 (1974)

37. Penrose, R., Rindler, W.: Spinors and Space-Time: Spinor and Twistor Methods in Space-Time Geometry, vol. 2. Cambridge University Press, Cambridge (1986)

38. Meissner, K.A., Nurowski, P., Ruszczycki, B.: Structures in the microwave background radiation. Proc. R. Soc. A 469(2155), 20130116 (2013)

39. DeAbreu, A., Contreras, C., Scott, D.: Searching for concentric low variance circles in the cosmic microwave background. JCAP 12, 031 (2015)

40. Gurzadyan, V.G., Penrose, R.: CCC and the Fermi paradox. Eur. Phys. J. Plus 131, 11 (2016)

41. An, D., Meissner, K.A., Nurowski, P.: Ring-type structures in the Planck map of the CMB. Month. Notices Roy. Astron. Soc. 473, 3251-3255, arXiv:1510.06537 astro-ph.CO (2018)

42. Gasperini, M., Veneziano, G.: The pre-big bang scenario in string cosmology. Phys. Rep. 373, 1-212 (2003) 
43. Independent personal communications from James Peebles and from Joseph Silk

44. Károlyházy, F.: Gravitation and quantum mechanics of macroscopic bodies. Nuovo Cim. A 42, 390 (1966)

45. Károlyházy, F.: Gravitation and quantum mechanics of macroscopic bodies. Magyar Fizikai Polyoirat 12, 24 (1974)

46. Percival, I.C.: Quantum spacetime fluctuations and primary state diffusion. Proc. R. Soc. Lond. A 451, 503-513 (1995)

47. Penrose, R.: General-relativistic energy flux and elementary optics. In: Hoffmann, B. (ed.) Perspectives in Geometry and Relativity, pp. 259-274. Indiana University Press, Bloomington (1966)

48. Kramer, D., Stephani, H., MacCallum, M.H.A., Herlt, E.: Exact Solutions of Einstein's Field Equations. VEB Deutscher Verlag der Wissenschaften, Cambridge University Press, Cambridge (1980)

49. Huneau, C., Luk, J.: High-frequency backreaction for the Einstein equations under polarized U(1) symmetry, arXiv:1706.09501 [gr-qc] (2017)

50. Cresswell, J., vonHausegger, S., Jackson, A.D., Hao, L., Naselsky, P.: On the time lags of the LIGO signals. arXiv:1706.04191v1 [astro-ph.IM]. (2017) Accessed 13 June 2017

51. Sabín, C., Bruschi, D.E., Ahmadi, M., Fuentes, I.: Phonon creation by gravitational waves. N. J. Phys. 16, 085003 (2014)

52. Sabín, C., Kohlrus, J., Bruschi, D.E., Fuentes, I.: EPJ Quantum Technol. 3, 8 (2016) 